Ciência Florestal, Santa Maria, v. 22, n. 4, p. 853-864, out.-dez., 2012

ISSN 0103-9954

\title{
ESTIMATIVA DO VOLUME TOTAL DE MADEIRA EM ESPÉCIES DE EUCALIPTO A PARTIR DE IMAGENS DE SATÉLITE LANDSAT
}

\author{
TOTAL WOOD VOLUME ESTIMATION OF Eucalyptus SPECIES BY \\ IMAGES OF LANDSAT SATELLITE
}

\author{
Elias Fernando Berra ${ }^{1}$ Catize Brandelero ${ }^{2}$ Rudiney Soares Pereira ${ }^{3}$ \\ Elódio Sebem ${ }^{4}$ Laura Camila de Godoy Goergen ${ }^{5}$ \\ Ana Caroline Paim Benedetti ${ }^{6}$ Diogo Belmonte Lippert ${ }^{7}$
}

\begin{abstract}
RESUMO
Modelos relacionando respostas espectrais com parâmetros biofísicos visam estimar variáveis, tais como volume de madeira, sem a necessidade de avaliações constantes em campo. Objetivou-se desenvolver modelos estimadores de volume de madeira a partir de imagens TM do Landsat 5, com base em dados de inventário florestal regional. A imagem foi georreferenciada e convertida para imagem reflectância espectral. Em seguida, foram geradas as imagens índice NDVI (índice de vegetação da diferença normalizada) e SR (razão simples). Os valores das reflectâncias das bandas (TM1, TM2, TM3 e TM4) e dos índices (NDVI e SR) foram relacionados com os volumes de madeira. As maiores correlações com volume foram com os índices NDVI e SR. A seleção de variáveis foi feita pelo método Stepwise, o qual retornou três modelos de regressão como significativos para explicar a variação em volume. Por fim, selecionou-se o modelo com melhor ajuste (volume $=-830,95+46,05 \times(\mathrm{SR})+107,47 \times(\mathrm{TM} 2)$ ), o qual foi aplicado sobre a imagem Landsat onde os pixels passaram a representar o volume estimado $\mathrm{em} \mathrm{m}^{3} / \mathrm{ha}$ nas unidades de produção de Eucalyptus sp. Este modelo, significativo ao nível de $95 \%$ de confiança, explica $68 \%$ da variação de volume de madeira.
\end{abstract}

Palavras-chave: parâmetros biofísicos; reflectância; índices de vegetação.

\section{ABSTRACT}

Models relating spectral answers with biophysical parameters aim estimate variables, such as wood volume, without the necessity of frequent field measurements. The objective was to develop models to estimate wood volume by Landsat 5 TM images, supported by regional forest inventory data. The image was georeferenced and converted to spectral reflectance. After, the images-index NDVI (Normalized Difference Vegetation Index) and SR (Simple Ratio) was generated. The reflectance values of both spectral bands

1. Engenheiro Florestal, Mestrando do Programa de Pós-graduação em Sensoriamento Remoto, Universidade Federal do Rio Grande do Sul, Av. Bento Gonçalves, 9500, Campus do Vale, Bairro Agronomia, Caixa Postal 15044, CEP 91501-970, Porto Alegre (RS). Bolsista do CNPq. eliasberra@yahoo.com.br

2. Engenheira Florestal, $\mathrm{Dr}^{\mathrm{a}}$, Professor Adjunto do Departamento de Engenharia Rural, Centro de Ciências Rurais, Universidade Federal de Santa Maria, Av. Roraima, 1000, CEP 97105-900, Santa Maria (RS). catizebrandelero@gmail.com

3. Engenheiro Florestal, Dr., Professor Titular do Departamento de Engenharia Rural, Centro de Ciências Rurais, Universidade Federal de Santa Maria, Av. Roraima, 1000, CEP 97105-900, Santa Maria (RS). rudiney@smail.ufsm.br

4. Engenheiro Florestal, Dr., Professor do Colégio Politécnico da UFSM, Universidade Federal de Santa Maria, Av. Roraima, 1000, Prédio 70, CEP 97105-900, Santa Maria (RS). elodiosebem@politecnico.ufsm.br

5. Acadêmica do Curso de Engenharia Florestal, Universidade Federal de Santa Maria, Av. Roraima, 1000, CEP 97105-900, Santa Maria (RS). lauragoergen@yahoo.com.br

6. Engenheira Florestal, $\mathrm{Dr}^{\mathrm{a}}$, Professor Adjunto, Universidade Federal do Pampa, campus São Gabriel, Rua Gabriel Coimbra Meyer, 123-257, CEP 97300-000, São Gabriel (RS). anabenedetti@unipampa.edu.br

7. Engenheiro Florestal, Doutorando do Programa de Pós Graduação em Engenharia Florestal, Centro de Ciências Rurais, Universidade Federal de Santa Maria, Av. Roraima, 1000, CEP 97105-900, Santa Maria (RS). diogolippert@hotmail.com

Recebido para publicação em 24/03/2011 e aceito em 09/08/2011 
(TM1, TM2, TM3 e TM4 ) and indices (NDVI and SR) was related with the wood volume. The biggest correlation with volume was with the NDVI and SR indices. The variables selection was made by Stepwise method, which returned three regression models as significant to explain the variation in volume. Finally, the best fitted model was selected (volume $=-830,95+46,05 \times(\mathrm{SR})+107,47 \times(\mathrm{TM} 2)$ ), which was applied on the Landsat image where the pixels had started to represent the estimated volume in $\mathrm{m}^{3} / \mathrm{ha}$ on the Eucalyptus sp. production units. This model, significant at $95 \%$ confidence level, explains $68 \%$ of the wood volume variation.

Keywords: biophysical parameters; reflectance; vegetation indices.

\section{INTRODUÇÃO}

Segundo Watzlawick et al. (2009), as consequências da redução das florestas no ciclo do carbono vêm criando uma demanda de desenvolvimento de métodos não destrutivos para a determinação de biomassa e volume de madeira, elemento importante na modelagem dos ciclos biogeoquímicos.

As determinações de volume de madeira podem ser trabalhosas, demoradas e onerosas, e podem ser realizadas por meio de métodos diretos ou indiretos. Pelo método direto, as árvores são cortadas e seus componentes medidos (diâmetro em diferentes alturas e altura), e pelo método indireto são utilizadas equações alométricas ou utilização de técnicas de sensoriamento remoto, para realizar as estimativas (SANQUETTA e BALBINOT, 2004).

Com os atuais avanços tecnológicos na área de sensoriamento remoto e os desenvolvimentos relacionados aos avanços no processamento das imagens, tem se obtido outra maneira de caracterização da estrutura das florestas e, consequentemente de sua biomassa e/ou volume. Para a obtenção de estimativas, o fundamento é associar aos dados de radiância/reflectância, provenientes de imagens de satélite, dados medidos a campo (SOUZA e PONZONI, 1998; ROSENQVIST et al., 2003).

Estimativas de parâmetros biofísicos da vegetação, como volume de madeira, consistem em um importante uso do sensoriamento remoto (PETERSON e RUNNING, 1989). Para a estimativa de volume de madeira florestal em escala local e regional, são necessários dados provenientes de instrumentos sensores. Nesse sentido, podem ser utilizados dados do sensor TM da série de satélites Landsat como já demonstrado por diversos autores (FAZAKAS et al., 1999; HÄME et al., 1997; KRANKINA et al., 2004; TOMPPO et al., 2002; TURNER et al., 2004).

$\mathrm{Na}$ imagem, os alvos podem ser caracterizados pela reflectância em cada uma das bandas espectrais ou através de índices. Para o estudo de vegetação, segundo Ponzoni e Shimabukuru (2007), os índices de vegetação são transformações radiométricas amplamente utilizadas.

Pearson e Miller (1972) notaram a existência de uma correlação linear negativa entre o fator de reflectância e a vegetação verde no comprimento de onda do vermelho, e uma correlação linear positiva no infravermelho próximo. Assim, a partir do final da década de 60 , pesquisadores começaram a desenvolver vários índices de vegetação, com o intuito de caracterizar alguns parâmetros biofísicos da vegetação.

Brandão et al. (2005) explicam que, embora sejam encontrados mais de 50 índices de vegetação na literatura, muitos estudos comprovam a eficácia dos dois índices mais comumente usados que são: a Razão Simples (SR) e o Índice de Vegetação da Diferença Normalizada (NDVI). O índice SR foi definido por Jordan (1969) a partir da divisão da reflectância referente à região do infravermelho próximo pela região do vermelho. Já o índice NDVI, proposto por Kriegler et al. (1969) e Rouse et al. (1973), é obtido pela Equação 1.

$$
\text { NDVI }=\frac{\text { IVP-V }}{\text { IVP+V }} \quad \text { Equação (1) }
$$

Em que: IVP $=$ região espectral do infravermelho próximo; $\mathrm{V}=$ região espectral do vermelho.

Os índices de vegetação baseados em cocientes (SR e NDVI, entre outros) trabalham com as bandas espectrais do vermelho e infravermelho próximo em virtude do comportamento da vegetação nestas bandas espectrais. A vegetação vigorosa de espécies folhosas absorve radiação na zona do vermelho $(0,6$ a $0,7 \mu \mathrm{m})$ e reflete a maior parte da energia incidente na região do infravermelho próximo $(0,7$ a $1,1 \mu \mathrm{m})$ (PINILLA, 1995). Assim o volume de madeira pode ser estimado pelos índices de vegetação em questão. 
O Estado do Rio Grande do Sul (RS) tem dado especial atenção ao levantamento de sua cobertura florestal e seus parâmetros biofísicos, sendo o primeiro da Federação a elaborar Inventário Florestal (IF) detalhado, entre os anos de 1999 e 2001. Segundo a Associação Gaúcha de Empresas Florestais-AGEFLOR (2009), o setor silvícola movimenta atualmente $\mathrm{R} \$ 4,5$ bilhões e pretende dobrar o atual faturamento e alcançar no Estado a soma de $\mathrm{R} \$ 10$ bilhões nos próximos 10 anos. $\mathrm{O}$ plantio de acácia-negra, eucalipto e pinus com fins comerciais no RS já ocupa mais de 560 mil hectares, o que equivale a $1,9 \%$ da superfície do Estado. Com os novos investimentos de expansão, se forem confirmados, estima-se que a área plantada chegaria a um total de 900 mil hectares no prazo de 10 anos, colocando o Estado como o segundo polo florestalindustrial de todo o Brasil. Com isso, existe uma demanda muito grande por soluções tecnológicas de baixo custo que permitam acompanhar a dinâmica das áreas florestais, sua cobertura e/ou exploração bem como, a disponibilidade de estimativa de matéria-prima para diferentes segmentos do setor florestal.

Mandallaz (1993) comenta que há uma demanda crescente para usar dados de inventários disponíveis, tanto regionais como nacionais, para fins de estimação de parâmetros a níveis locais.

Nesse contexto, este trabalho, centraliza o enfoque no domínio e entendimento de metodologia para construção de modelos matemáticos associados aos dados de sensores remotos, com o objetivo de permitir aplicações em estimativas de volume de madeira como suporte inclusive a estudos futuros relacionados à biomassa. Assim, pretende-se responder se é possível realizar estimativas de volume de madeira, utilizando-se técnicas de sensoriamento remoto orbital aliados aos dados de campo obtidos em inventário regional, especificamente o inventário florestal contínuo do Estado do Rio Grande do Sul, em povoamentos de Eucalyptus sp.

\section{MATERIAL E MÉTODO}

O presente estudo localiza-se na região leste do Estado de Rio Grande do Sul, onde estão implantados, em plantações de Euclyptus sp., Unidades Amostrais (U.A.) do Inventário Florestal (IF). A Figura 1 mostra a localização das U.A. e a respectiva numeração.

De acordo com a classificação de Köppen, o Estado do RS fica dividido em duas áreas climáticas, "Cfa" e "Cfb", sendo que, a variedade "b" se restringe ao planalto basáltico superior e ao escudo Sul-Rio-Grandense, enquanto que as demais áreas pertencem à variedade "a" (MORENO, 1961). Na Figura 1, a área representada pela letra (a) possui um clima "Cfb" e uma altitude média de $100 \mathrm{~m}$; a área (b) um clima "Cfa" e altitude média de $240 \mathrm{~m}$; a área (c) um clima "Cfa" e altitude média de $30 \mathrm{~m}$.

A primeira etapa do trabalho consistiu em verificar a disponibilidade de imagens da série Landsat para associá-las com o período em que foi realizado o IF (1999 a 2001); esta etapa teve o objetivo de identificar cenas coincidentes com a localização das áreas das U.A. dentro deste intervalo de tempo; selecionaram-se as cenas com ausência ou pouca cobertura de nuvens.

Em gerenciador de banco de dados, fez-se uma consulta aos dados do IF onde se informavam as seguintes restrições para retorno da consulta: floresta plantada, do gênero Eucalyptus sp., latitude e longitude (referentes à área da cena do sensor analisado) e data. Os dados levantados pelo IF nas florestas plantadas continham basicamente as informações de diâmetro e altura (com as quais se estima o volume) a localização da U.A. e o gênero. Informações complementares como procedência (semente ou jardim clonal), espaçamento, densidade de plantas, índice de área foliar, entre outras, não foram levantadas.

$\mathrm{Na}$ data onde havia uma cena de satélite disponível, pesquisou-se a ocorrência de medidas de U.A. do IF naquele mês, um mês antes e um mês depois da passagem do satélite, retornando assim, quais seriam as U.A. medidas na área da cena num intervalo de três meses que contemplassem o gênero Eucalyptus sp. Assim, obtiveram-se dados de U.A. no mesmo mês, um mês antes e um mês depois da data da imagem.

As medidas das U.A. utilizadas neste trabalho foram realizadas durante período do inverno, época de menor crescimento da planta no ano. Desta forma, o intervalo escolhido, três meses, representa um incremento em volume de madeira muito baixo, ou próximo de zero.

A imagem utilizada neste estudo foi a do satélite Landsat 5 sensor TM, com resolução espacial de $30 \mathrm{~m}$; na data de passagem de 23/06/2000 na órbita 221 ponto 081 ; nas bandas TM1 (azul), TM2 (verde), TM3 (vermelho) e TM4 (infravermelho próximo); ângulo de elevação solar de $25,4543^{\circ}$.

Para realizar o georreferenciamento da 


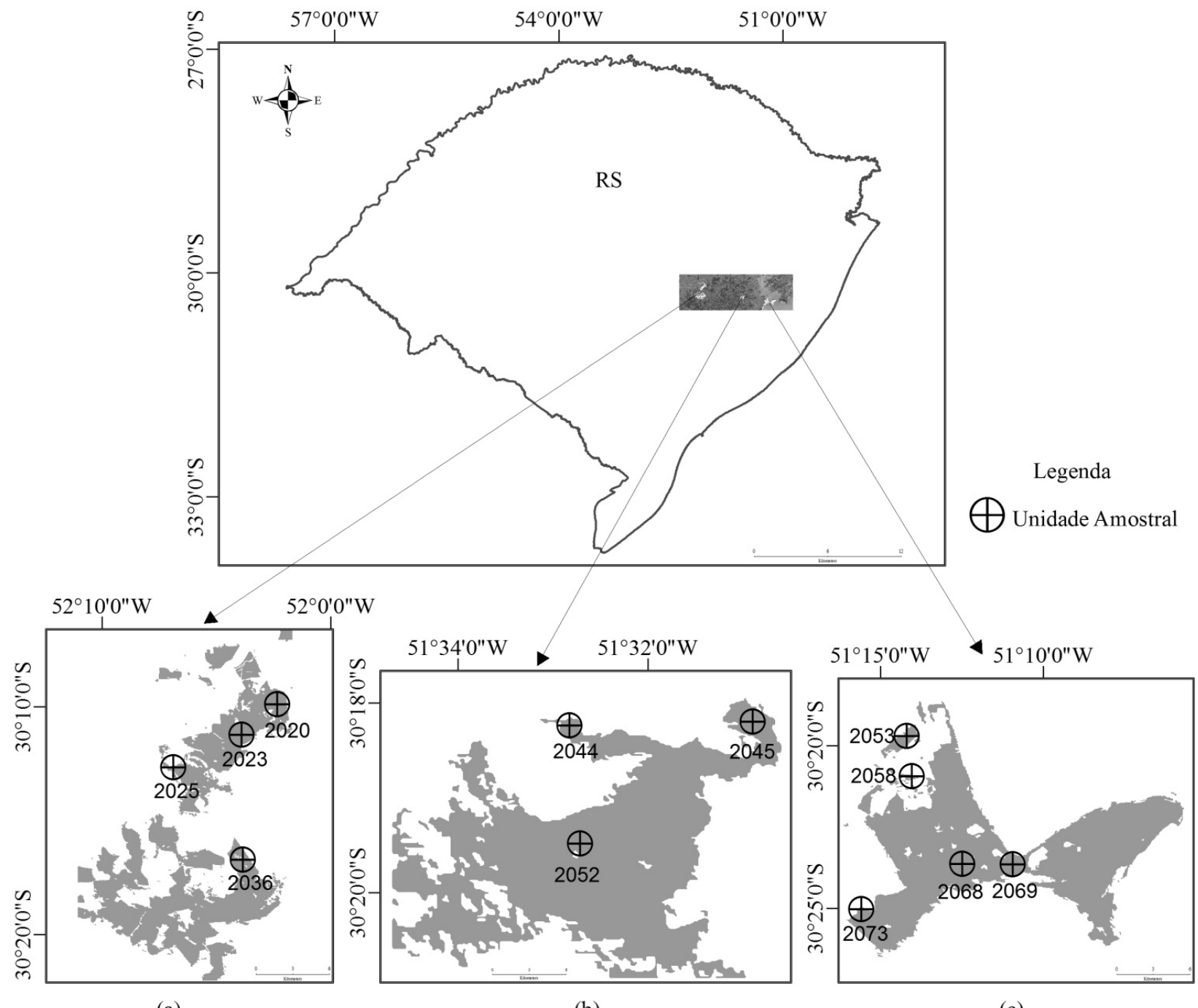

(a)

(b)

(c)

FIGURA 1: Mapa de localização das Unidades Amostrais (UA) em plantios de Eucalyptus sp.; a) Plantio com as U.A. 2020, 2023, 2025 e 2036; b) Plantio com as U.A. 2044, 2045 e 2052; c) Plantio com as U.A. 2053, 2058, 2068, 2069 e 2073.

FIGURE 1: Location map of the sample units in Eucalyptus sp.; a) Planting with the sample units 2020, 2023, 2025 and 2036; b) Planting with the sample units 2044, 2045 and 2052; c) Planting with the sample units 2053, 2058, 2068, 2069 and 2073.

imagem usou-se a carta topográfica do Exército, Barra do Ribeiro (Folha SH-22-Y-B-II-4). O método utilizado para fazer a reamostragem dos pixels foi o vizinho mais próximo.

Para associar os dados inventariados com a reflectância da imagem orbital, realizou-se a conversão dos números dos pixels para radiância/ reflectância, segundo a metodologia descrita por Gürtler et al. (2005).

As U.A. do Inventário Florestal Contínuo do Rio Grande do Sul são do formato circular com um raio de $13,84 \mathrm{~m}$. A partir das coordenadas geodésicas referentes ao centro das U.A., foi delimitada uma área de igual forma e tamanho. Os valores de reflectância foram obtidos de duas maneiras: a primeira quando a U.A. estivesse contida em um único pixel, onde o valor de reflectância correspondeu ao próprio pixel; e a segunda quando a U.A. abrangeu dois ou quatro pixels fez-se a média aritmética.

O tratamento estatístico foi realizado inicialmente com a análise de correlação entre o volume de madeira e a reflectância das bandas TM1, TM2, TM3 e TM4 e os índices NDVI e SR.

Para definir quais dados das imagens que melhor estimam o volume de madeira, utilizou-se análise de regressão. 
Para gerar os modelos de estimativa de volume de madeira $\left(\mathrm{m}^{3} / \mathrm{ha}\right)$, foram testados os valores de reflectância das U.A. nas bandas do azul, do verde, do vermelho e do infravermelho próximo, e os índices de vegetação NDVI e SR, sendo que, todos entraram no modelo como variáveis independentes. Os dados oriundos do IF (volume de madeira/ha) entraram como variáveis dependentes. Utilizou-se o método de eliminação de variáveis Stepwise (passo a passo), onde foram selecionadas somente as variáveis que mais contribuíam para o modelo proposto. Com a utilização destes modelos foi possível realizar o mapeamento do volume de madeira para os plantios de Eucalyptus sp. na área de estudo.

\section{RESULTADOS E DISCUSSÃO}

Na Tabela 1, são apresentadas as 12 U.A., cujo IF foi realizado entre maio e julho de 2000.

Ao aplicar a metodologia descrita por Gürtler et al.(2005) para transformação de ND em reflectância corrigida em relação ao espalhamento atmosférico, obtiveram-se as equações para as quatro bandas, que são apresentadas na Tabela 2 .

Ao aplicar as equações da Tabela 2 gerouse uma imagem de reflectância de superfície, da qual se obtiveram as informações apresentadas na Tabela 3, cujos dados serviram de entrada para o processamento estatístico.

Na Tabela 3 são apresentados os valores de reflectância de superfície nas bandas do TM 1, 2, 3 e 4, bem como os valores dos índices de vegetação, referentes às 12 Unidades Amostrais inventariadas. Observa-se um comportamento espectral típico das florestas de folhosas, com respostas baixas na região do visível, aumentando no infravermelho próximo.

Na região que compreende os comprimentos de onda do infravermelho próximo $(0,7 \mu \mathrm{m}$ a $1,3 \mu \mathrm{m})$, existe uma pequena absorção desta radiação por parte do Eucalyptus sp., consequentemente, temos uma região de alta reflectância de IVP dessa vegetação, resultado da interação da energia incidente com a estrutura do mesófilo (estrutura

TABELA 1: Unidades Amostrais selecionadas do Inventário Florestal Contínuo do Rio Grande do Sul. TABLE 1: Sample units selected from Rio Grande do Sul state Continuous Forest Inventory.

\begin{tabular}{ccccl}
\hline NPAR & Data & Latitude & Longitude & Espécie \\
\hline 2020 & $03 / 05 / 00$ & $-30^{\circ} 09^{\prime} 53^{\prime \prime}$ & $-52^{\circ} 02^{\prime} 19^{\prime \prime}$ & Eucalyptus $\mathrm{sp}$. \\
2023 & $03 / 05 / 00$ & $-30^{\circ} 11^{\prime} 13^{\prime \prime}$ & $-52^{\circ} 03^{\prime} 53^{\prime \prime}$ & Eucalyptus $\mathrm{sp}$. \\
2025 & $03 / 06 / 00$ & $-30^{\circ} 12^{\prime} 37^{\prime \prime}$ & $-52^{\circ} 07^{\prime} 02^{\prime \prime}$ & Eucalyptus $\mathrm{sp}$. \\
2036 & $03 / 06 / 00$ & $-30^{\circ} 16^{\prime} 40^{\prime \prime}$ & $-52^{\circ} 03^{\prime} 55^{\prime \prime}$ & Eucalyptus $\mathrm{sp}$. \\
2044 & $03 / 07 / 00$ & $-30^{\circ} 18^{\prime} 09^{\prime \prime}$ & $-51^{\circ} 32^{\prime} 46^{\prime}$ & Eucalyptus $\mathrm{sp}$. \\
2045 & $03 / 07 / 00$ & $-30^{\circ} 18^{\prime} 11^{\prime \prime}$ & $-51^{\circ} 31^{\prime} 14^{\prime \prime}$ & Eucalyptus $\mathrm{sp}$. \\
2052 & $03 / 07 / 00$ & $-30^{\circ} 19^{\prime} 29^{\prime \prime}$ & $-51^{\circ} 32^{\prime} 43^{\prime \prime}$ & Eucalyptus $\mathrm{sp}$. \\
2053 & $03 / 05 / 00$ & $-30^{\circ} 19^{\prime} 33^{\prime \prime}$ & $-51^{\circ} 14^{\prime} 19^{\prime \prime}$ & Eucalyptus $\mathrm{sp}$. \\
2058 & $03 / 05 / 00$ & $-30^{\circ} 20^{\prime} 55^{\prime \prime}$ & $-51^{\circ} 14^{\prime} 02^{\prime \prime}$ & Eucalyptus $\mathrm{sp}$. \\
2068 & $03 / 05 / 00$ & $-30^{\circ} 23^{\prime} 38^{\prime \prime}$ & $-51^{\circ} 12^{\prime} 29^{\prime \prime}$ & Eucalyptus $\mathrm{sp}$. \\
2069 & $03 / 05 / 00$ & $-30^{\circ} 23^{\prime} 39^{\prime \prime}$ & $-51^{\circ} 10^{\prime} 56^{\prime \prime}$ & Eucalyptus $\mathrm{sp}$. \\
2073 & $03 / 05 / 00$ & $-30^{\circ} 24^{\prime} 58^{\prime \prime}$ & $-51^{\circ} 15^{\prime} 36^{\prime \prime}$ & Eucalyptus $\mathrm{sp}$. \\
\hline
\end{tabular}

Em que: NPAR = Número da U.A.; DATA = Data do inventário da U.A.

TABELA 2: Equações utilizadas para gerar a imagem com informação de reflectância de superfície.

TABLE 2: Equation used for generate image with surface reflectance information.

\begin{tabular}{cc}
\hline Banda & Equação \\
\hline TM 1 & Reflectância $1=(0,00280 \times \mathrm{ND})-0,07854$ \\
TM 2 & Reflectância $2=(0,00555 \times \mathrm{ND})-0,05554$ \\
TM 3 & Reflectância $3=(0,00473 \times \mathrm{ND})-0,04256$ \\
TM 4 & Reflectância $4=(0,00773 \times \mathrm{ND})-0,03092$ \\
\hline
\end{tabular}


Berra, E. F. et al.

TABELA 3: Volume de madeira, reflectâncias de superfície e índices de vegetação das Unidades Amostrais. TABLE 3: Wood volume, reflectance and, vegetation indexes of the sample units.

\begin{tabular}{|c|c|c|c|c|c|c|c|}
\hline \multirow{2}{*}{ NPAR } & \multirow{2}{*}{ Volume $\left(\mathrm{m}^{3} / \mathrm{ha}\right)$} & \multicolumn{4}{|c|}{ Reflectância } & \multicolumn{2}{|c|}{ Índices de Vegetação } \\
\hline & & TM1 & TM2 & TM3 & TM4 & NDVI & SR \\
\hline 2020 & 477,633 & 0,044 & 0,021 & 0,009 & 0,372 & 0,950 & 39,342 \\
\hline 2023 & 675,640 & 0,034 & 0,019 & 0,007 & 0,283 & 0,955 & 43,533 \\
\hline 2025 & 296,202 & 0,038 & 0,022 & 0,011 & 0,297 & 0,931 & 27,788 \\
\hline 2036 & 261,055 & 0,036 & 0,022 & 0,010 & 0,330 & 0,941 & 33,096 \\
\hline 2044 & 666,425 & 0,035 & 0,022 & 0,006 & 0,262 & 0,959 & 47,521 \\
\hline 2045 & 687,207 & 0,039 & 0,028 & 0,009 & 0,448 & 0,959 & 47,397 \\
\hline 2052 & 643,900 & 0,035 & 0,020 & 0,009 & 0,302 & 0,941 & 32,814 \\
\hline 2053 & 233,588 & 0,047 & 0,029 & 0,018 & 0,271 & 0,878 & 15,385 \\
\hline 2058 & 183,172 & 0,043 & 0,033 & 0,022 & 0,326 & 0,875 & 15,013 \\
\hline 2068 & 583,222 & 0,039 & 0,025 & 0,010 & 0,294 & 0,936 & 30,284 \\
\hline 2069 & 504,107 & 0,039 & 0,023 & 0,011 & 0,329 & 0,933 & 28,726 \\
\hline 2073 & 377,408 & 0,036 & 0,032 & 0,013 & 0,281 & 0,914 & 22,192 \\
\hline & $\overline{\mathrm{X}}$ & 0,039 & 0,025 & 0,011 & 0,316 & & \\
\hline
\end{tabular}

Em que: NPAR $=$ Número da Unidade Amostral; Volume $\left(\mathrm{m}^{3} / \mathrm{ha}\right)=$ Volume de madeira com casca, estimado por hectare; TM1, TM2, TM3 e TM4 = Reflectância nas bandas do azul, verde, vermelho e infravermelho próximo, respectivamente; NDVI = Índice de Vegetação por Diferença Normalizada; SR = Razão Simples.

celular). Isso ocorre, pois a infiltração de água diminui os espaços vazios do interior das folhas, diminuindo o espalhamento da energia incidente $\mathrm{e}$ aumentando a transmitância (WATZLAWICK et al., 2009). Já na região do vermelho $(0,63$ a $0,69 \mu \mathrm{m})$ ocorre a máxima absorção, a absorção desta região é feita pela clorofila. A região do azul $(0,45-0,52 \mu \mathrm{m})$ também é caracterizada por uma forte absorção causada pelos carotenóides e pelas clorofilas. Uma relativa diminuição de absorção acontece entre as bandas do azul e do vermelho, isto é, na região do verde $\left(0,54 \mu_{\mathrm{m}}\right)$ do espectro eletromagnético (JENSEN, 2009).

Diante do exposto, observa-se na Tabela 3, na região do visível, que a maior média de reflectância está na banda do azul (TM1) e não na região do verde (TM2). Na região do visível, quando o alvo for uma folha verde e sadia, espera-se que a banda do verde apresente a maior reflectância, de acordo com o parágrafo acima. Contudo, quando se trabalha com imagem de satélite em uma área de floresta, a resposta espectral da vegetação desta área é soma da resposta espectral dos vários alvos componentes do ambiente (além das folhas da planta) mais a interferência atmosférica podendo, assim, o comportamento espectral da vegetação na imagem apresentar-se diferente do comportamento espectral de uma folha verde e sadia. Conforme Shimabukuro (1987), os dados de radiância coletados pelos sensores remotos dependem das características de registro do sensor e das características espaciais e espectrais dos alvos. A radiação registrada pelo sensor é a soma da radiância de todos os materiais dentro do campo de visada instantânea e da contribuição da atmosfera.

$\mathrm{Na}$ Tabela 3 observa-se que os maiores valores de NDVI, referentes às Unidades Amostrais 2023, 2044 e 2055 correspondem aos maiores valores de volume. Já os menores valores de NDVI nas Unidades Amostrais 2053 e 2058 correspondem aos menores valores de volume. Na SR, como era de se esperar, o comportamento é semelhante, uma vez que o NDVI apresenta apenas um realce do SR. As U.A. 2023, 2044 e 2055 apresentam os maiores valores de SR, estes também correspondem aos maiores valores de volume. Esse comportamento é explicado por Ponzoni e Shimabukuro (2007) e Meneses e Madeira Netto (2001), estes informam que normalmente a relação esperada entre a cobertura vegetal e os valores dos índices NDVI e SR sejam diretamente proporcionais; essa explicação é aceitável para os casos onde a cobertura vegetal não apresenta densidade tal capaz de promover a ocorrência dos pontos de saturação em qualquer 
uma das bandas espectrais em questão e ainda as sombras não ocasionem oscilações inesperadas nos valores de fatores de reflectâncias, criando comportamento de respostas anômalas.

Também, pode-se observar na Tabela 3 que as relações entre o volume e os índices de vegetação não apresentam proporcionalidade direta em todas as U.A. observadas, indicando possíveis pontos de saturação da resposta espectral das bandas e dos índices. Observa-se, por exemplo, que a U.A. 2052 apresenta um volume de madeira de $643,9 \mathrm{~m}^{3} / \mathrm{ha}(\mathrm{NDVI}=0,941$ e $\mathrm{SR}=32,814)$, já a U.A. 2036 apresenta um menor volume de madeira, $261,0 \mathrm{~m}^{3} / \mathrm{ha}$, e o mesmo valor de NDVI $(0,941) \mathrm{e}$ um maior valor de SR $(33,096)$.

\section{Análise estatística}

Primeiramente são apresentados os resultados obtidos na análise de correlação entre as variáveis independentes (reflectância nas quatro bandas e os índices de vegetação) e a variável dependente (volume de madeira) obtidas com o emprego de função linear. Logo após, são apresentados os resultados oriundos da análise de regressão com os modelos que melhor explicam a dependência entre as variáveis.

A matriz de correlação, Tabela 4, mostra o grau de dependência entre as variáveis. A variável dependente apresenta as maiores correlações positivas com as variáveis espectrais $\operatorname{SR}(0,82)$ e NDVI $(0,79)$, em destaque na Tabela 4 . A banda espectral que apresenta maior valor absoluto de correlação com a variável dependente é a banda
TM3 $(-0,78)$. De maneira geral, as bandas do visível (TM1, TM2 e TM3) respondem inversamente ao aumento de volume, enquanto a banda do infravermelho próximo (TM4) se correlaciona diretamente.

Pesquisadores têm demonstrado desde 1960 que existe uma relação direta entre a resposta espectral no infravermelho próximo e as variáveis relacionadas com biomassa. Por outro lado, também tem sido demonstrado que existe uma relação inversa entre a resposta no visível, particularmente no vermelho, e as variáveis relacionadas com biomassa de plantas (JENSEN, 2009; PEARSON e MILLER, 1972).

$\mathrm{Na}$ Tabela 4, observa-se uma relação inversamente proporcional entre o aumento do volume e a energia refletida na porção do espectro eletromagnético correspondente ao vermelho (TM3). O aumento da absorção da energia nesta faixa do espectro eletromagnético é consequência direta do aumento da quantidade de pigmentos fotossintetizantes (KNIPLING, 1970; THOMAS e GAUSMAN, 1977; FONSECA, 2004). No infravermelho próximo (TM4) a quantidade de energia refletida aumenta com o aumento do volume de madeira, isto pode ser devido ao aumento na quantidade de espaços intercelulares por área da copa. Estes espaços intercelulares promovem aumento nas reflexões e refrações sucessivas da radiação eletromagnética no interior do mesófilo das várias camadas de folhas (WOOLLEY, 1971; THOMAS e GAUSMAN, 1977; FONSECA, 2004).

TABELA 4: Coeficientes de correlação entre o volume de madeira e as variáveis digitais obtidos por meio da função linear, para plantios de Eucalyptus sp.

TABLE 4: Coefficients of correlation between wood volume and the digital variables obtained by linear function, for Eucalyptus sp. plantings.

\begin{tabular}{lccccccc}
\hline & Volume $\left(\mathrm{m}^{3} / \mathrm{ha}\right)$ & TM1 & TM2 & TM3 & TM4 & NDVI & SR \\
\hline Volume $\left(\mathrm{m}^{3} / \mathrm{ha}\right)$ & 1 & & & & & & \\
TM1 & $-0,52$ & 1 & & & & & \\
TM2 & $-0,53$ & 0,52 & 1 & & & & \\
TM3 & $-0,78$ & 0,70 & 0,81 & 1 & & & \\
TM4 & 0,17 & 0,21 & 0,10 & $-0,02$ & 1 & & \\
NDVI & 0,79 & $-0,63$ & $-0,76$ & $-0,95$ & 0,30 & 1 & \\
SR & 0,82 & $-0,52$ & $-0,66$ & $-0,87$ & 0,35 & 0,94 & 1 \\
\hline
\end{tabular}

Em que: volume $\left(\mathrm{m}^{3} / \mathrm{ha}\right)=$ volume de madeira $\mathrm{em} \mathrm{m}^{3} / \mathrm{ha}$; TM1, TM2, TM3 e TM4 = reflectância nas bandas do azul, verde, vermelho e infravermelho próximo, respectivamente; NDVI = índice de vegetação de diferença normalizada; $\mathrm{SR}=$ razão simples. 
O valor da correlação entre a TM4 com volume $(\mathrm{r}=0,17)$, apesar de indicar a existência de relação direta entre as variáveis, é muito baixo. $\mathrm{Na}$ Tabela 3, percebe-se que o aumento de volume não acompanha o aumento da reflectância em todas as U.A. Por exemplo, a U.A. 2044 apresenta o terceiro maior volume observado $\left(666,4 \mathrm{~m}^{3} / \mathrm{ha}\right)$ e a menor reflectância na TM4 $(0,262)$. Assim, nessa U.A., na TM4, era de se esperar que este maior volume aumentasse o espaço intercelular por área de copa e aumentasse também a energia refletida, contudo, a energia não foi refletida na proporção esperada. Sabe-se que a arquitetura do dossel influencia na resposta espectral da vegetação, e quando o efeito do sombreamento na cena é bastante pronunciado pode ocorrer uma diminuição da resposta espectral no infravermelho próximo. Ponzoni e Shimabukuro (2009), explicam que, quando a cobertura vegetal atinge uma alta densidade, as sombras podem ocasionar oscilações inesperadas nos valores de reflectância das bandas espectrais, criando comportamento de respostas anômalas, ou seja, menores valores de reflectância aparecerão em florestas mais densas.

Assim, as maiores correlações estão associadas às faixas espectrais que compreendem os comprimentos de onda do infravermelho próximo (TM4) e vermelho (TM3), comprovadas por meio dos índices que utilizam esta relação (NDVI e SR). Segundo Ponzoni e Shimabukuro (2007), a fundamentação da proposição dos índices de vegetação reside no comportamento antagônico da reflectância da vegetação nas regiões espectrais do visível e infravermelho próximo (IVP). Portanto, como regra geral, tem-se que, por um lado, quanto maior for a densidade da cobertura vegetal em uma determinada área, menor será a reflectância na região do visível devido a maior oferta de pigmentos fotossintetizantes. Por outro lado, maior será a reflectância verificada na região do IVP devido ao espalhamento múltiplo da radiação eletromagnética nas diferentes camadas de folhas.

Resultados encontrados no presente estudo são similares aos encontrados pelos autores abaixo:

Canavesi e Ponzoni (2007), relacionando variáveis dendrométricas de Eucalyptus sp. com imagem TM do Landsat 5, encontraram correlação negativa entre as bandas do visível (TM1, TM2 e TM3) e o volume de madeira ( $\left.\mathrm{m}^{3} / \mathrm{ha}\right)$ para uma classe de iluminação plana.

Pinheiro et al. (2009) trabalharam com imagem TM do Landsat 5 na estimativa de biomassa aérea no cerrado (cerrado stricto sensu e cerradão). Os autores obtiveram índices de correlação negativos para as bandas do visível (TM1, TM2 e TM3) e positivos para a banda do infravermelho (TM4) tanto no cerrado stricto sensu como cerradão. Os autores obtiveram índices de correlação positivos para o NDVI de 0,32 no cerrado stricto sensu e 0,51 no cerradão. Para o índice SR as correlações foram de 0,53 no cerradão e 0,33 no cerrado stricto sensu, também positivas. É importante destacar que os dois índices de vegetação apresentaram relações consistentes do ponto de vista físico, uma vez que todas as correlações foram positivas.

Ponzoni e Shimabukuro (1998) estudaram a relação entre índices de vegetação e parâmetros biométricos de plantios de Eucalyptus urophylla e Eucalyptus camaldulensis utilizando imagens TM do Landsat 5. Os autores encontraram correlação positiva de 0,33 entre NDVI e volume de madeira $\left(\mathrm{m}^{3} / \mathrm{ha}\right)$.

Diferentes satélites também foram testados em estudos similares ao aqui apresentado. Gebreslasie (2008) investigou a utilidade do satélite ASTER (resolução espacial de $15 \mathrm{~m}$ ) na estimativa de parâmetros biofísicos, entre eles o volume de madeira, em plantações de Eucalyptus sp. na África do Sul. As correlações das bandas espectrais com o volume $\left(\mathrm{m}^{3} / \mathrm{ha}\right)$ foram negativas nas bandas do verde e do vermelho do espectro visível $(-0,08$ na banda verde e $-0,32$ na banda do vermelho) e positiva para a banda do infravermelho próximo $(0,20)$. Com os índices de vegetação, o autor encontrou correlação de 0,37 para NDVI e 0,17 para o SR, ambas positivas; Fernandes et al. (2011), usaram imagens AVNIR-2 do ALOS para estimar parâmetros biofísicos em plantações de eucaliptos. Os autores encontraram correlações positivas entre o volume de madeira com NDVI $(r=0,15)$ e com SR $(r=0,16)$.

\section{Ajuste e seleção de equação para o volume}

$\mathrm{Na}$ Tabela 5 são apresentados os cinco modelos de regressão de estimadores de volume de madeira, sendo que os modelos 1, 2 e 3 são resultado do Stepwise; no modelo 4 foi inserido o índice SR, já no modelo 5 o índice inserido foi o NDVI. Os modelos 1 e 2 apresentaram os melhores ajustes, com coeficientes de determinação ajustados de 0,68 e 0,67 , respectivamente, e os menores coeficientes de variação $(\mathrm{CV})$. De modo geral todas as equações são compostas com pelo menos um dos índices de vegetação (NDVI ou SR), como era de se esperar, uma vez que estas variáveis apresentaram as maiores correlações com a variável volume, expostas na 
Tabela 4.

Os valores para $\mathrm{R}^{2} \quad\left(\mathrm{R}_{\text {Ajust }}^{2}\right)$ para os modelos da Tabela 5 variaram de 0,61 a 0,68 . Vários autores empregaram modelos para estimar parâmetros biofísicos de coberturas florestais e encontraram valores similares; Xavier et al. (1998) estimando Índice de Área Foliar (IAF) de plantações de eucaliptos a partir de dados Landsat 5/TM encontraram um modelo com $\mathrm{R}^{2}$ de 0,69 ; Thenkabail et al. (2003), utilizando sensores multiespectrais para estimar volume de madeira e dados dos sensores IKONOS e Landsat 7/ ETM+ para espacializar o volume de madeira na área de estudo, encontraram valores de $\mathrm{R}^{2}$ variando de 0,34 a 0,69; Hall et al. (2005) também realizaram um trabalho com o mesmo contexto, obtendo $\mathrm{R}^{2}$ de 0,69 para dados do sensor Landsat 7/ ETM+; empregando dados do HyMap, Schlerf et al. (2005) encontraram $\mathrm{R}^{2}$ de 0,79 na estimativa do volume; Canavesi et al. (2010) estimaram volume de madeira em plantios de Eucalyptus spp. utilizando dados Hyperion EO-1 e encontraram valores de $\mathrm{R}^{2}$, ajustados nos modelos, que variaram de 0,621 a 0,704, sendo que, em 3 dos 4 modelos incluíram índices de vegetação; Gebreslasie (2008), relacionando volume com imagens ASTER $(15 \mathrm{~m})$, encontrou um modelo de regressão para estimar o volume $\left(\mathrm{m}^{3}\right) \operatorname{com~} \mathrm{R}^{2}$ de 0,30 para povoamentos jovens de Eucalyptus sp. e $\mathrm{R}^{2}$ de 0,27 para povoamentos maduros; Watzlawick et al. (2009) estimando parâmetros biofísicos em floresta com araucária utilizando IKONOS II encontraram $\mathrm{R}^{2}$ ajustados variando de 0,57 a 0,71 .

A escolha do modelo, para espacialização do volume na imagem da área de estudo deste trabalho, baseou-se na análise das estatísticas usualmente empregadas ( $\left.F, \mathrm{R}_{\text {Ajust }}^{2}, \mathrm{~S}_{\mathrm{yx}}, \mathrm{CV}\right)$. A equação 1 , por apresentar melhor ajuste ( $\mathrm{F}$ significativo, maior
$\mathrm{R}^{2}{ }_{\text {Ajust? }}$ menor $\mathrm{S}_{\mathrm{yx}}$ e menor $\mathrm{CV}$ ), foi selecionada e utilizada para estimar o volume dos plantios florestais na imagem em estudo.

Os modelos aqui selecionados podem ser reaplicados para plantações florestais do gênero Eucalyptus sp., nas condições aqui consideradas, pois os resultados podem variar para outros tipos de vegetação, estrutura, bem como composição de espécies e para outros métodos de determinação das variáveis. A aparência da cobertura vegetal em uma imagem orbital é consequência de um processo complexo que envolve muitos parâmetros e fatores ambientais. Para Ponzoni e Shimabukuro (2007), a reflectância espectral de dosséis é influenciada por fatores de natureza geométrica (visada), espectral (propriedades espectrais dos elementos da vegetação, principalmente das folhas e do solo) e biofísica (IAF e Distribuição Angular das Folhas- DAF); Lu et al. (2004) em suas análises mencionam que os elementos do ecossistema influenciam a resposta espectral e, portanto, afetam diretamente os modelos ajustados para a obtenção de dados biofísicos estimados por meio de satélites.

\section{Quantificação do volume na imagem}

Utilizando o modelo ajustado número 1 , da Tabela 5 , foi gerada uma nova imagem, em que cada pixel passou a representar a quantidade estimada de volume de madeira por hectare. A nova imagem pode ser visualizada na Figura 2. A região da Figura 2 corresponde à área (c) da Figura 1.

Pela análise visual do mapa apresentado, observou-se uma homogeneização dos valores de volume após o fatiamento dos valores estimados, suavizando a variabilidade espacial verificada nos valores espectrais das Unidades Amostrais e dos valores de volume.

TABELA 5: Modelos de regressão testados e ajustados para estimar a variável volume de madeira em plantios de Eucalyptus sp.

TABLE 5: Regression models tested and adjusted for estimate wood volume on Eucalyptus sp. plantings.

\begin{tabular}{c|c|c|c|c|c|c}
\hline $\mathrm{N}$ & $\begin{array}{c}\text { Variável } \\
\text { Dependente }\end{array}$ & $\begin{array}{c}\text { Modelo de } \\
\text { Regressão }\end{array}$ & $\mathrm{F}$ & $\mathrm{R}_{\text {Ajust }}^{2}$ & $\mathrm{~S}_{\mathrm{yx}}$ & $\mathrm{CV}(\%)$ \\
\hline 1 & Vol & $-830,95+46,05 .(\mathrm{SR})+107,47 .(\mathrm{TM} 2)$ & $12,86^{*}$ & 0,68 & 106,35 & 22,83 \\
\hline 2 & Vol & $-565,21+136,03 .(\mathrm{TM} 2)-90,89 .(\mathrm{TM} 3)+36,25(\mathrm{SR})$ & $8,36^{*}$ & 0,67 & 108,95 & 23,40 \\
\hline 3 & Vol & $-8234,38+118,79 .(\mathrm{TM} 2)-97,84 .(\mathrm{TM})+34,95 .(\mathrm{NDVI})$ & $7,04^{*}$ & 0,62 & 116,15 & 24,94 \\
\hline 4 & Vol & $-109,93+32,78 .(\mathrm{SR})$ & $18,5^{*}$ & 0,61 & 117,38 & 25,19 \\
\hline 5 & Vol & $-8011,82+35,27 .(\mathrm{NDVI})$ & $18,06^{*}$ & 0,61 & 118,31 & 25,40 \\
\hline
\end{tabular}

Em que: * = Significativo ao nível de $95 \%$ de probabilidade; $\mathrm{N}=$ número do modelo; $\mathrm{Vol}=$ volume de $\mathrm{madeira}\left(\mathrm{m}^{3} / \mathrm{ha}\right)$; $\mathrm{R}^{2}{ }_{\text {Ajust }}=$ Coeficiente de determinação ajustado; $\mathrm{S}_{\mathrm{yx}}=$ Erro padrão da estimativa; CV\%= Coeficiente de variação em \%; $\mathrm{F}=$ Valor de $\mathrm{F}$ da análise de variância. 


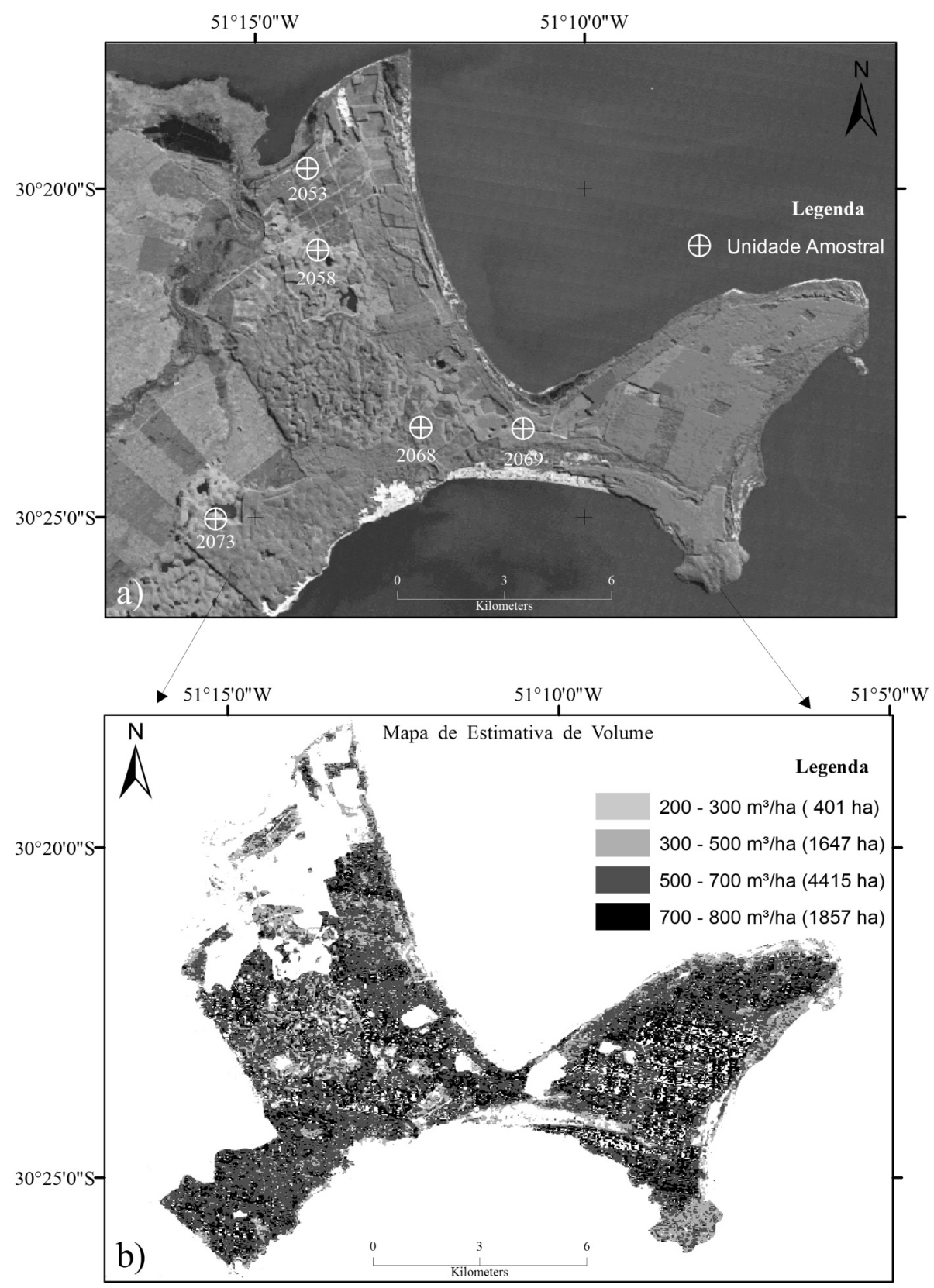

FIGURA 2: Em a) imagem com plantio de Eucalyptus sp. e localização das Unidades Amostrais 2053, 2058, 2068, 2069 e 2073. Em b) imagem temática resultante da modelagem de volume de madeira estimado por hectare, distribuído em classes, na unidade de produção de Eucalyptus sp.

FIGURE 2: On a) image with Eucalyptus sp. plantings and location of the sample units 2053, 2058, 2068, 2069 and 2073. On b) thematic image resulting of modeling of estimated wood volume per hectare, separated at classes, on the Eucalyptus sp. unit production.

Assim, pode-se estimar diretamente sobre a imagem o volume de madeira, onde cada pixel representa uma área de $900 \mathrm{~m}^{2}$. Ou ainda, podem-se delimitar polígonos com áreas de interesse e calcular a média do volume estimado e ajustar para a área total, resultando no volume total de uma determinada unidade de produção.

\section{CONCLUSÕES}

Dentre as bandas espectrais, os dados da banda 3 do sensor TM do Landsat 5 foram os que apresentaram a maior correlação com o volume de madeira. Os dois índices de vegetação estudados apresentaram maiores correlações com o volume em 
relação às bandas espectrais individualmente.

Os índices NDVI e SR mostraram-se como bons estimadores de volume de madeira; existe alta correlação positiva entre volume de madeira e os valores de NDVI e SR para plantios de Eucalyptus sp. na área de estudo.

As regressões múltiplas geradas com as bandas espectrais e os índices de vegetação explicam o volume de madeira de uma forma melhor que as regressões simples geradas com estas mesmas variáveis, individualmente.

A equação com melhor ajuste estatístico possibilitou a geração de um mapa temático com as estimativas de volume de madeira de Eucalyptus sp. na área de estudo.

\section{REFERÊNCIAS BIBLIOGRÁFICAS}

AGEFLOR - Associação Gaúcha de Empresas florestais. A próxima década das florestas 20/03/2009. Disponível em: < (www.ageflor.com. br)> Acesso em: 12 de fevereiro de 2011.

BRANDÃO, Z. N. et al. Determinação de índices de vegetação usando imagens de satélite para uso em agricultura de precisão. In: CONGRESSO BRASILEIRO DE ALGODÃO, 5., 2005, Salvador. Anais.... Campina Grande: CNPA, 2005.

CANAVESI, V. et al. Estimativa de volume de madeira em plantios de Eucalyptus spp. utilizando dados hiperespectrais e dados topográficos. Revista Árvore, Viçosa, v. 34, n. 3, p. 539-549, maio/jun. 2010.

CANAVESI, V., PONZONI, F.J. Relações entre variáveis dendrométricas de plantios de Eucalyptus sp. e valores de FRB de superfície de imagens do sensor TM/Landsat 5. In: SIMPÓSIO BRASILEIRO DE SENSORIAMENTO REMOTO, 13., 2007, Florianópolis. Anais.... INPE: São José dos Campos, 2007, p. 1619-1625.

FAZAKAS, Z. et al. Regional forest biomass and wood volume estimation using satellite data and ancillary data. Agricultural and Forest Meteorology, v. 98, p. 417-425, dez. 1999.

FERNANDES, D. H. F. M. et al. Uso de imagens ALOS para estimar parâmetros biofísicos em plantações de eucaliptos. In: SIMPÓSIO BRASILEIRO DE SENSORIAMENTO REMOTO, 15., 2011, Curitiba. Anais.... INPE: São José dos Campos, 2011, p. 1674-1671.

FONSECA, E. L. Desenvolvimento de modelo da disponibilidade de fitomassa aérea para formações campestres naturais a partir de dados espectrais orbitais e agrometeorológicos. 2004. Tese (Doutorado em Sensoriamento Remoto) Instituto Nacional de Pesquisas Espaciais, São José dos Campos, 2004.

GEBRESLASIE, M. T. The estimation of eucalyptus plantation forest structural attributes using medium and high spatial resolution satellite imagery. 2008. Tese (Doctor of Philosophy in Applied Environmental Science) - University of KwaZulu-Natal Pietermartizburg, South Africa, 2008.

GÜRTLER, S. et al. Planilha eletrônica para o cálculo da reflectância em imagens TM e ETM+ LANDSAT. Revista Brasileira de Cartografia, v. 57, p. 162167, ago. 2005.

HALL, R. J. et al. Modeling forest stand structure attributes using Landsat ETM + data: Application to mapping imaging spectrometer data. Remote Sensing of Environment, v.95, n.1, p.177-194, Mar. 2005.

HÄME, T. et al. A new methodology for estimation of biomass of conifer-dominated boreal forest using NOAA AVHRR data. International Journal of Remote Sensing, v. 18, p. 3211-3243, Jan. 1997.

JENSEN, J. R. Sensoriamento remoto do ambiente: uma perspective em recursos terrestres. São José dos Campos: Parêntese, 2009. 598 p.

JORDAN, C. F. Derivation of leaf-area index from quality of light on the forest floor. Ecology, v. 50, p. 663-666, July 1969.

KNIPLING, E. B. Physical and physiological basis for the reflectance visible and near infrared radiation from vegetation. Remote Sensing of Environment, v.1, n.3, p.155-159, Feb. 1970.

KRANKINA, O. N. et al. Carbon stores, sinks, and sources in forests of North-western Russia: Can we reconcile forest inventories with remote sensing results? Climatic Change, v. 67, p.257-272, Dec. 2004.

KRIEGLER, F. J. et al. Preprocessing transformations and their effects on multispectral recognition. In: INTERNATIONAL SYMPOSIUM ON REMOTES SENSING OF ENVIRONMENT, 6., 1969, Michigan, USA. Proceedings...Michigan: University of Michigan, 1969. p.97-131. Disponível em: < (http:// adsabs.harvard.edu//abs/1969rse..conf...97K)> Acesso em: 04 de maio de 2010.

LU, D. et al. Relationship between forest stand parameters and Landsat TM spectral responses in the Brazilian Amazon Basin. Forest Ecology and Management, Amsterdan, v. 198, n.1-3, p. 149-167, Jan./Mar. 2004. 
MANDALLAZ, D. Geostatistical methods for double sampling schemes: application to combined forest inventory. Technical report, ETH Zürich, chair of forest inventory and planning, 133 p., 1993.

MENESES, P. R., MADEIRA NETTO, J. S. Sensoriamento remoto: reflectância de alvos naturais. Brasília: UNB-Embrapa Cerrados, 2001. $262 \mathrm{p}$.

MORENO, J. A. Clima do Rio Grande do Sul. Porto Alegre: Secretaria da Agricultura. 1961. 41 p.

PEARSON, R. L.; MILLER, L. D. Remote mapping of standing crop biomass for estimation of the productivity of the short-grass Prairie. In: INTERNATIONAL SYMPOSIUM ON REMOTE SENSING OF ENVIRONMENT, 8., Ann Arbor, 1972. Proceedings. Ann Arbor: ERIM, 1972, pp. 1357-1381.

PETERSON, D.L., RUNNING, S.W. Aplications in Forest science and management. In: Asrar, G. Theory and applications of optical remote sensing. New York, John Wiley, 1989. Cap. 10, p. 429-473.

PINHEIRO, E. S. et al. Imagens Landsat e QuickBird são capazes de gerar estimativas precisas de biomassa aérea de Cerrado? In: SIMPÓSIO BRASILEIRO DE SENSORIAMENTO REMOTO, 14., 2009, Natal. Anais... INPE:São José dos Campos, 2009, p. 29132920.

PINILLA, C. Elementos de teledetección. Madrid: RA-MA, 1995. $313 \mathrm{p}$.

PONZONI, F. J., SHIMABUKURO, Y. E.. Relação entre índices de vegetação e parâmetros biométricos de plantios de Eucalyptus urophylla e Eucalyptus camaldulensis em Cachoeira do Manteiga (MG). Revista Árvore, Viçosa, v. 22, n. 3, p. 357-366, jul./ set. 1998.

PONZONI, F. J., SHIMABUKURU, Y.E. Sensoriamento remoto no estudo da vegetação. São José dos Campos: A. Silva Vieira, 2007. 150 p. PONZONI, F. J., SHIMABUKURU, Y.E. Sensoriamento remoto no estudo da vegetação. São José dos Campos: A. Silva Vieira, 2009. 127 p. ROSENQVIST, A. et al. A review of remote sensing technology in support of the Kyoto protocol. Environmental Science \& Policy, v. 6, p. 441-455, Oct. 2003.

ROUSE, J. W. et al. Monitoring vegetation systems in the Great Plains with ERTS. In: ETRS SYMPOSIUM, 3., 1973, Washington D.C., USA. Proceedings... Washington D.C.: NASA SP-351, 1973. p.309-317.

SANQUETTA， C. R; BALBINOT, C. R. R.
Metodologias para determinação de biomassa florestal. In: Fixação de carbono: atualizações, projetos e pesquisas. Curitiba. 2004. p. 77-94.

SCHLERF, M. et al. Remote sensing of forest biophysical variables using HyMap imaging spectrometer data. Remote Sensing of Environment, v. 95, n. 1, p.177-194, Mar. 2005.

SHIMABUKURO, Y. E. Shade Images Derived from Linear Mixing Models of Multispectral Measurements of Forested Areas. 1987. (Ph.D. Dissertation) - Colorado State University, Fort Collins, 1987.

SOUSA, C. L., PONZONI, F. J. Avaliação de índices de vegetação e de bandas TM/Landsat para estimativa de volume de madeira em floresta implantada de Pinus spp. In: SIMPÓSIO BRASILEIRO DE SENSORIAMENTO REMOTO, 9., 1998, Santos, SP. Anais.... INPE: São José dos Campos, 1998, v.13, p. 1725-1732.

THENKABAIL, P. S. et al. Detecting floristic structure and pattern across topographic and moisture gradients in a mixed species Central African forest using IKONOS and Landsat-7 ETM+ images. International Journal of Applied Earth Observation and Geoinformation, v. 4, n. 3, p.255270, June 2003.

THOMAS, J. R., GAUSMAN, H. W. Leaf reflectance vs. leaf chlorophyll and carotenoid concentrations for eight crops. Agronomy Journal, v. 69, n. 5, p. 799802, 1977.

TOMPPO, E. et al. Simultaneous use of Landsat-TM and IRS-1c WiFS data in estimating large area tree stem volume and aboveground biomass. Remote Sensing of Environment, v. 82, p. 156-171, Sept. 2002.

TURNER, D. P. et al. Monitoring forest carbon sequestration with remote sensing and carbon cycle modeling. Environmental Management, v. 33, p. 457-466, Aug. 2004.

WATZLAWICK, L. F. et al. Estimativa de biomassa e carbono em floresta com araucaria utilizando imagens do satélite IKONOS II. Ciência Florestal, Santa Maria, v. 19, p.169-181, abr./jun. 2009.

WOOLLEY, J.T. Reflectance and transmitance of light by leaves. Plant Physiology, v.47, n.3, p. 656662, May 1971.

XAVIER, A. C. et al. Estimativa de IAF de plantações de eucaliptos a partir de dados TM/Landsat. In: SIMPÓSIO BRASILEIRO DE SENSORIAMENTO REMOTO, 9., 1998, Santos. Anais... INPE: São José dos Campos, 1998. p. 1585-1596. 